Gynecologic and

Obstetric Investigation
Gynecol Obstet Invest 2018;83:234-240

DOI: $10.1159 / 000487319$
Received: November 13, 2017

Accepted after revision: January 31, 2018

Published online: March 14, 2018

\title{
Evaluating Colposcopy with Dynamic Spectral Imaging During Routine Practice at Five Colposcopy Clinics in Wales: Clinical Performance
}

\author{
Srividya Budithia Richard Peevor ${ }^{a} \quad$ David Pugh $^{b} \quad$ Emmanouil Papagiannakis ${ }^{c}$ \\ Amanda Durman $^{c}$ Nassera Banu $^{d}$ Aderemi Alalade $^{\mathrm{e}}$ Simon Leeson $^{\mathrm{a}}$ \\ ${ }^{a}$ Department of Obstetrics and Gynecology, Ysbyty Gwynedd, Bangor, UK; ${ }^{b}$ Department of Obstetrics and

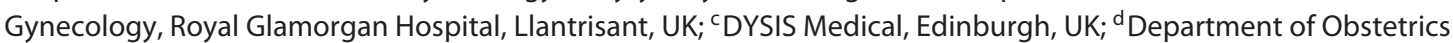 \\ and Gynecology, Ysbyty Glan Clwyd, Boddelwyddan, UK; ${ }^{e}$ Department of Obstetrics and Gynecology, Wrexham \\ Maelor Hospital, Wrexham Maelor, UK
}

\author{
Keywords \\ Cervical intraepithelial neoplasia $\cdot$ Cervical intraepithelial \\ neoplasia 2-3 - Colposcopy
}

\begin{abstract}
Background: The diagnostic accuracy of colposcopy is poor for detecting precancerous cervical lesions. Objectives: We assessed the performance of colposcopy for identifying cervical intraepithelial neoplasia grade 2 or worse (CIN2+), before and after including a dynamic spectral imaging (DSI) map that quantifies and maps acetowhitening to assist subsequent biopsy of suspicious lesions. Methods: Four hundred and twenty-five women were examined at a multi-center setting in Wales, of which 393 women were included in the final analysis. Results: For all referrals, the sensitivity of conventional colposcopy for histologically confirmed CIN2+ was $51.5 \%$, the specificity was $92.0 \%$, the positive predictive value was $56.7 \%$, and the negative predictive value (NPV) was $90.4 \%$. With the incorporation of the DSI map in predicting CIN2+, these became 84.8, 61.5, 30.8, and 95.3\% respectively. The increase of sensitivity was statistically significant $(p<0.001)$. For the 236 women having colposcopy after lowgrade (LG) cytology, with the incorporation of DSI, the sensitivity for CIN2+ increased from 27.3 to $86.4 \%(p<0.001)$
\end{abstract}

and the NPV from 92.6 to $97.8 \%$. Conclusions: Colposcopy with DSI results in improved sensitivity to detect CIN2+ and maintains a high NPV for all referrals and especially for those with LG referral cytology.

(c) 2018 S. Karger AG, Basel

\section{Introduction}

Colposcopy has a poor ability to detect cervical intraepithelial neoplasia grade 2 or worse (CIN2+) as studies suggest a sensitivity for CIN2+ of 49-61\% [1-6]. Gage et al. [7] reported that $30 \%$ of CIN3 + was missed at initial colposcopy in the Atypical Squamous Cells of Undetermined Significance (ASC-US) and Low Grade Triage Study. However, performance is related to the prevalence of the abnormality, and future service changes may lead to a further deterioration of sensitivity. The incidence of CIN has declined within 3 years of implementation of human papillomavirus (HPV) vaccination in Australia [8]. The need for treatment for CIN is expected to be reduced by at least $50 \%$ if there is an uptake of vaccination of $80 \%$ of the eligible population, which is being achieved in the UK [911]. Introduction of HPV-based screening may have a similar effect. A post-implementation report of HPV-

\section{KARGER}

(c) 2018 S. Karger AG, Basel

E-Mail karger@karger.com

www.karger.com/goi
Simon Leeson

Department of Obstetrics and Gynecology

Ysbyty Gwynedd

Bangor, LL57 2PW (UK)

E-Mail simon.leeson@wales.nhs.uk 
based screening in Italy revealed that $11.4 \%$ of colposcopy referrals yielded a diagnosis of CIN2+ following cytology triage with ASC-US or worse [12]. This compares to $29.8 \%$ of all colposcopy referrals having CIN2+ for cytologybased screening [13]. As a consequence of this combination of changes, we would anticipate less CIN2+ and less operator exposure in recognizing colposcopic features of high-grade (HG) CIN. The ability for colposcopy to predict HG disease is likely to decline. HPV-based screening may increase the rate of referral of cases for colposcopic assessment by as much as $100 \%$ for HPV positive women without any form of triage for women over 30 years of age compared to women referred to colposcopy with ASC-US or worse cytology [14]. The sensitivity of colposcopy is paramount in identifying smaller proportions of referred cases with pre-malignant lesions. Ideally this should not be at the cost of lower specificity but a sacrifice in specificity is to be expected. In clinical practice, a drop in specificity may not mean that more biopsies are required.

There is a prospect of improving the objectivity and performance of colposcopy with a new technology. Dynamic spectral imaging (DSI) involves the measurement of acetowhitening and modeling of a color-coded map to assist the identification of HG CIN by localizing and grading the severity of any cervical lesion. DYSIS (DYSIS Medical Ltd., Edinburgh, UK) is a digital video colposcope that integrates the proprietary DSI technology to evaluate the blanching effect of applying acetic acid to cervical epithelium. It produces a quantitative measurement of the rate, extent, and duration of acetowhitening. The color-coded map (DSI map) produced is overlaid on the live image of the cervix to help the clinician determine the presence, location, and grade of any lesion and assist in selecting sites for biopsy.

Studies on DSI-assisted colposcopy have shown promising results with an improved sensitivity for the detection of CIN2+ $[4,5,15]$. The National Institute for Health and Clinical Excellence commissioned an independent health economics study and then published a recommendation that DSI colposcopy is a clinically cost-effective method of cervical assessment [16].

The performance of DSI colposcopy on low grade (LG) cytological referrals is only known from a sub-analysis of a multi-center study [17]. We report a prospective service evaluation of the DSI colposcope at colposcopy clinics in Wales to measure the detection of HG cervical disease in a multi-center environment, during use in routine practice. The objective was to compare the performance of colposcopy with the DSI map for all, and specifically for LG referrals, against conventional assessment for the detection of CIN2+.

\section{Materials and Methods}

Five colposcopy clinics in Wales agreed to participate. In total, 425 women were enrolled as new colposcopy referrals from May 2014 to May 2015. Cases were recruited from consecutive routine colposcopy appointments at Cardiff and Vale, the Royal Glamorgan Hospital, Wrexham Maelor Hospital, Ysbyty Glan Clwyd, and Ysbyty Gwynedd. Of these 425 women, 6 cases were non-cervical examinations, leaving 419 cervical colposcopies for evaluation. $\mathrm{Pa}$ tients included were those with abnormal cervical cytology referred from Cervical Screening Wales (CSW) to colposcopy. Women were referred with a single HG cervical cytology sample; after 2 samples reported as LG dyskaryosis; after 3 samples reported as borderline change in squamous cells; or a combination of 2 LG and borderline change in squamous cells. HPV triage was not used in Wales at the time of service evaluation. Women were also included if referred from primary care with an abnormal appearing cervix and post-coital bleeding. All colposcopists had 4 clinical sessions (20 examinations) of training to familiarize themselves with the DSI colposcope, prior to the evaluation.

Non-cervical disease (e.g., vulval and vaginal referrals) and pregnant women were excluded. Data were entered on and collected for analysis on the all-Wales colposcopy database (Canisc; https:// canisc.cymru.nhs.uk/Canisc.htm). Colposcopists were permitted to take an excisional or diagnostic punch biopsies as per their normal personal practice and as per CSW standard operating procedures.

\section{Clinical Procedures}

All women had acetic acid applied to the cervix and had a colposcopic examination with a DSI colposcope to incorporate a standard colposcopy followed by DSI mapping of the cervix. Colposcopist assessment of the disease grade on each case was recorded prior to and then with the use of the DSI map.

Schiller's test was optional but if it was used then iodine was applied after the DSI map was viewed. Saline or a green filter was used as desired. Patients were subsequently managed as per CSW standard operating procedures. Areas selected for biopsy with conventional colposcopy were selected prior to seeing the DSI map. Additional areas suggesting biopsy revealed by the DSI map had biopsies as the colposcopist felt appropriate for each patient. The colposcopist was always free to interpret and over-ride the DSI map impression as would be the case in clinical practice. The DSI color-coded map was considered as suggestive for HG CIN and positive for biopsy if red, yellow or white areas were superimposed upon the cervical image. However, biopsies were not separately labeled as DSI-directed or as standard colposcopy-directed biopsies due to a short-term requirement to outsource some pathology slides during the course of service evaluation.

The following data items were recorded for each case: age, smoker status, referral reason (cytology LG/HG or other), squamocolumnar junction seen, extent of lesion, colposcopic assessment pre-DSI map, colposcopic assessment post-DSI map, histology, and management plan.

\section{Data Analysis}

All analyses were performed per patient; in this design of incremental examination (first conventional assessment, followed by the addition of the DSI map as an adjunct), each patient served as her own control. For each patient, we recorded the colposcopist's assessment prior to seeing the DSI map readings, then the assessment com- 
Table 1. Baseline characteristics of patients across the 5 clinics

\begin{tabular}{|c|c|c|c|c|c|c|}
\hline Number & 67 & 109 & 22 & 55 & 166 & 419 \\
\hline Age, years, mean & 34.1 & 32.8 & 34.5 & 32.6 & 32.8 & 33.1 \\
\hline Age, years, median & 31 & 30 & 33 & 29 & 28 & 29 \\
\hline $25-75$ percentiles, years & $26-42$ & $26-40$ & $28-41$ & $24-41$ & $24-43$ & $25-41$ \\
\hline Current smoker, $\%$ & 35.8 & 17.4 & 31.8 & 29.1 & 32.5 & 28.6 \\
\hline SCJ seen*, \% & 95.5 & 93.6 & 95.5 & 81.8 & 94.0 & 92.6 \\
\hline
\end{tabular}

SCJ, Squamocolumnar junction; CV, Cardiff and Vale; RGH, Royal Glamorgan Hospital; WMH, Wrexham Maelor Hospital; YGC, Ysbyty Glan Clwyd; YG, Ysbyty Gwynedd.

* Information not reported for $2.4 \%$ of the patients.

bining the colposcopist's findings and that of the DSI map. When either was HG, then the combined assessment was considered as HG, and if both were normal the combined assessment was considered as that of a normal cervix. Although conventional findings would influence the final colposcopic assessment, the sensitivity for detecting of HG CIN would be inevitably increased with the integration of the DSI map. Histologically confirmed disease severity of CIN2+ has been considered as the gold standard for analysis. For the assessment of the performance of colposcopy, we used a HG prediction as the correct indication for patients eventually found with CIN2+. Patients with no biopsy (including those with inadequate/unknown biopsy result) were considered as normal in the analysis, introducing inevitable bias in the calculations as the true status of non-biopsied tissue is unknown. This was preferred from excluding them from analyses, which would introduce significant bias in the specificity results, as the majority of these women are not expected to have HG disease. This effect was investigated in a secondary analysis that excluded women without a histology result.

The primary endpoint of the evaluation was to determine the sensitivity for CIN2+ when using the DSI map as compared to standard assessment for all women referred for colposcopy. The secondary endpoint was to determine the sensitivity for CIN2+ when using the DSI map as compared to the standard assessment for women referred with LG cytology.

McNemar's chi-square statistic with continuity correction was employed to test the distributional differences in the CIN2+ detection between the conventional assessment and the addition of the DSI map. The test was 1 -sided at $2.5 \%$ level of statistical significance. The magnitude of the sensitivity excess with the DSI map (Test) over the conventional method (Reference) was illustrated by the corresponding 1-sided $97.5 \%$ CI of the proportional difference between them (Test-Reference). Statistical analysis was performed in Rversion 3.2.2 (www.cran.r-project.org) utilizing the "Exact $2 \times 2$ " package [18].

\section{Results}

The numbers, mean and median age, percentage of current smokers, and whether the squamocolumnar junction was seen (Table 1) were typical of the colpos- copy referral population in Wales. For instance, from Canisc data for all new colposcopy referrals in Wales between June 1, 2014 and May 31, 2015, the mean age of colposcopy patients at their first visit was 35 years and $31.4 \%$ of the colposcopy population were recorded as smokers.

The distribution of referral indications can be seen from Table 2. Sixty percent of women were referred with a LG cytological abnormality, consistent with allWales data [19]. There were no cases referred with suspected carcinoma or suspected cervical glandular intraepithelial neoplasia. The distribution of histopathology findings can be seen from Table 3 . Biopsies were taken from 243 women (58.0\%) and CIN2+ was found in $69(16.5 \%)$. The total number of biopsies was not recorded. Some colposcopists added all their punch biopsies into one pot for each patient and sometimes it was not clear if apparent multiple biopsies were from a single fragmented sample. To overcome this, we recorded the worst grade of biopsy for each patient. There was some variation in colposcopist practice in that Cardiff and Vale had the highest proportion of HG referrals (49.3\% compared to $27.9 \%$ overall) but the lowest biopsy rate $(47.8 \%$ compared to $58.2 \%$ overall [Tables $1-3])$

There were 26 patients with some data items missing (including 3 with CIN2+): 25 did not have their colposcopic assessment recorded and the biopsy result for one was missing. Therefore, 393 women have been included in the analysis of colposcopic performance, 236 of them with a LG cytology referral.

A total of 66 women had CIN2+ among the 393 women. Conventional assessment predicted 34 as HG, and with the addition of the DSI map another 22 women were included, achieving a total of 56. Among the 
Table 2. Distribution of patient referrals across the 5 clinics. Data are numbers of patients (\% of total)

\begin{tabular}{llllrrr}
\hline Indication & CV & RGH & WMH & YGC & YG & Overall \\
\hline LG smear & $26(38.8)$ & $47(43.1)$ & $20(90.9)$ & $36(65.5)$ & $121(72.9)$ & $250(59.7)$ \\
HG smear & $33(49.3)$ & $38(34.9)$ & $1(4.5)$ & $17(30.9)$ & $27(16.3)$ & $116(27.7)$ \\
Other & $8(11.9)$ & $24(22.0)$ & $1(4.5)$ & $2(3.6)$ & $18(10.8)$ & $53(12.6)$ \\
\hline
\end{tabular}

LG, low-grade; HG, high-grade; "other," referred with an abnormal appearing cervix or post-coital bleeding; CV, Cardiff and Vale; RGH, Royal Glamorgan Hospital; WMH, Wrexham Maelor Hospital; YGC, Ysbyty Glan Clwyd; YG, Ysbyty Gwynedd.

Table 3. Description of biopsies and outcomes across the 5 clinics. Data are numbers of patients (\% of total)

\begin{tabular}{|c|c|c|c|c|c|c|}
\hline Outcome & $\begin{array}{l}\text { CV } \\
(n=67)\end{array}$ & $\begin{array}{l}\text { RGH } \\
(n=109)\end{array}$ & $\begin{array}{l}\text { WMH } \\
(n=22)\end{array}$ & $\begin{array}{l}\text { YGC } \\
(n=55)\end{array}$ & $\begin{array}{l}\mathrm{YG}^{*} \\
(n=166)\end{array}$ & $\begin{array}{l}\text { Overall } \\
(n=418)\end{array}$ \\
\hline No biopsy & $35(52.2)$ & $48(44.0)$ & $10(45.5)$ & $18(32.7)$ & $64(38.6)$ & $175(41.8)$ \\
\hline No CIN & $18(26.9)$ & $36(33.0)$ & $5(22.7)$ & $9(16.4)$ & $47(28.3)$ & $115(27.4)$ \\
\hline CIN1 & $5(7.5)$ & $6(5.5)$ & $6(27.3)$ & $14(25.5)$ & $28(16.9)$ & $59(14.1)$ \\
\hline CIN2 & $2(3.0)$ & $6(5.5)$ & $1(4.5)$ & $4(7.3)$ & $17(10.2)$ & $30(7.2)$ \\
\hline CIN3 & $7(10.4)$ & $13(11.9)$ & $0(0.0)$ & $10(18.2)$ & $9(5.4)$ & $39(9.3)$ \\
\hline
\end{tabular}

* One patient is missing any information on biopsy and is not included in this table.

CV, Cardiff and Vale; RGH, Royal Glamorgan Hospital; WMH, Wrexham Maelor Hospital; YGC, Ysbyty Glan Clwyd; YG, Ysbyty Gwynedd.

Table 4. Description of correlation between assessments and biopsy. Data are numbers of women

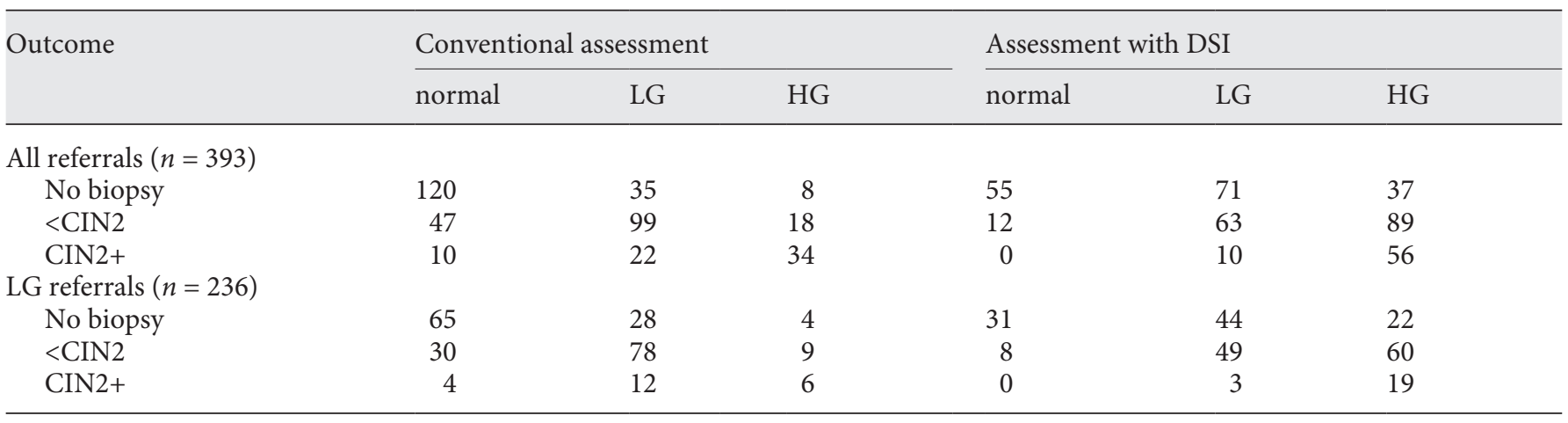

DSI, dynamic spectral imaging; CIN, cervical intraepithelial neoplasia; LG, low-grade; HG, high-grade.

236 women of the LG referral subgroup, conventional assessment predicted correctly 6 of the 22 women with CIN2+, and together with the DSI map another 13, achieving a total of 19 (Table 4). Eight women with a HG conventional colposcopic impression had no biopsies taken, of which 4 had been referred with LG cytology.

Within the sub-group of the 177 women (45\% of the total) with a conventional assessment of a normal cervix,
$57(32.2 \%)$ had biopsies taken and 10 were found to have CIN2+ (Table 4). Eight of these 10 women were considered as HG by incorporating the DSI map, and 2 were considered to be LG.

For all referrals, the sensitivity was $51.5 \%$, the specificity was $92.0 \%$, the positive predictive value (PPV) was $56.7 \%$ and the negative predictive value (NPV) was $90.4 \%$ for conventional colposcopy with histologically confirmed CIN2+. Following the inclusion of the DSI map, 
the corresponding percentages changed to $84.8,61.5$, 30.8 , and $95.3 \%$ (Table 5). After the inclusion of the DSI map, the sensitivity was increased by $33.3 \%$ (97.5\% CI $[22.5$ to $+\infty], p<0.001)$.

The impact of the DSI map addition was specifically analyzed in the sub-group of 236 women referred with LG cytology. For conventional colposcopy, the sensitivity was $27.3 \%$, the specificity was $93.9 \%$, the PPV was $31.6 \%$, and the NPV was $92.6 \%$ for histologically confirmed CIN2+. Following the inclusion of the DSI map, the corresponding percentages changed to $86.4,61.7,18.8$, and $97.8 \%$ (Table 5). In this sub-group, the increase in sensitivity with the addition of the DSI map was $59.1 \%$ ( $97.5 \%$ CI [ 38.7 to $+\infty], p<0.001)$.

For both sub-groups, the increase in sensitivity was at the cost of specificity that dropped by $30.5 \%$ (95\% CI [25.7-35.8], $p<0.001)$ and 32.2\% (95\% CI [26.1-39.0], $p<0.001)$, respectively.

In a secondary analysis on specificity, where patients with no biopsy result were not considered, for all types referrals ( 230 women), the specificity was $89.0 \%$ for conventional colposcopy and $45.7 \%$ with the inclusion of the DSI map. For patients with LG cytology (139 women), specificity was $92.3 \%$ for conventional colposcopy and $48.7 \%$ with the inclusion of the DSI map.

There were no cases of extension of cervical lesions onto the vagina.

\section{Discussion}

This prospective service evaluation of colposcopy with DYSIS at 5 clinics in Wales shows an improved sensitivity for colposcopy to identify patients with CIN2+ over conventional colposcopy, particularly for LG cytology referrals. The baseline patient characteristics appear representative of the all-Wales population of patients referred to colposcopy.

Our data agree with the sub-analysis of previous data in that we have demonstrated that the improvement of sensitivity is more pronounced among LG referrals, at a cost of specificity [17]. The sensitivity for CIN2+ with the use of the DSI map was $86 \%$ in the subgroup of women referred for colposcopy with LG cytology from our service evaluation, whereas the sensitivity of conventional colposcopy in this subgroup was poor (27\%). Louwers et al. [17] found a similar effect with the sub-analysis to detect CIN2+ for LG referrals of $82 \%$ as opposed to $44 \%$ for conventional colposcopy. In this subgroup, it could be that small volume HG CIN is difficult to iden-
Table 5. Clinical performance of colposcopic assessment before and with the DSI map in identifying high-grade (CIN2+) cervical pathology during routine colposcopy. Data are given in percentage $(95 \% \mathrm{CI})$

\begin{tabular}{lll}
\hline Outcome & $\begin{array}{l}\text { Conventional } \\
\text { assessment }\end{array}$ & $\begin{array}{l}\text { Assessment } \\
\text { with DSI }\end{array}$ \\
\hline \multicolumn{2}{l}{ All referrals $(n=393)$} & \\
$\quad$ Sensitivity & $51.5(39.0-63.9)$ & $84.8(73.4-92.1)$ \\
Specificity & $92.0(88.4-94.6)$ & $61.5(55.9-66.7)$ \\
PPV & $56.7(43.3-69.2)$ & $30.8(24.3-38.1)$ \\
NPV & $90.4(86.6-93.2)$ & $95.3(91.2-97.6)$ \\
LG smear referrals & $(n=236)$ & \\
Sensitivity & $27.3(11.6-50.4)$ & $86.4(64.0-96.4)$ \\
Specificity & $93.9(89.6-96.6)$ & $61.7(54.8-68.2)$ \\
PPV & $31.6(13.6-56.5)$ & $18.8(12.0-28.1)$ \\
NPV & $92.6(88.1-95.6)$ & $97.8(93.1-99.4)$ \\
\hline
\end{tabular}

DSI, dynamic spectral imaging; PPV, positive predictive value; NPV, negative predictive value; LG, low-grade.

tify colposcopically, or that colposcopic interpretation is biased by knowledge of LG referral cytology and so underestimates the severity of colposcopic appearances. These remain speculative as we did not record lesion size or the colposcopists' justification of colposcopic assessment.

Twenty-two of the 236 LG referrals had CIN2+ and 16 of the 22 had a normal or LG colposcopic opinion so that most HG abnormalities in the LG referral subgroup were identified by the DSI map alone. The improved yield of CIN2+ and earlier diagnosis results in a likelihood for less follow-up visits for repeat assessment and delayed treatment of previously missed lesions. Furthermore, a 5\% improved NPV to almost $98 \%$ for LG referrals means that a colposcopy with negative impression and negative DSI map is unlikely to be associated with a significant lesion. The increased sensitivity is at a cost of an increased false positive rate ( 1 - specificity) in the LG group from $6.1 \%$ for conventional colposcopy to $38.3 \%$ for DSI-assisted colposcopy (Table 5). This may lead to more biopsies without CIN2+ if colposcopists biopsied all DSI indications. However, use of the DSI map in our service evaluation did not seem to have an impact as our overall biopsy rate of $58 \%$ is consistent with contemporaneous biopsy rates at first attendance of $67.5 \%$ for all-Wales data [13] and 60\% from KC65 data for England [20].

Management during this service evaluation was as per standard clinical practice in Wales, and biopsies 
were not taken routinely unless an abnormality was suspected or for the assessment of HG referral cytology. This may have introduced ascertainment bias as some HG lesions may have been missed. This approach will tend to overestimate the sensitivity for both standard and DSI colposcopy as negative colposcopy cannot be confirmed in the absence of cervical biopsies. Although the exact reasons for not taking biopsies were not recorded for the 8 women with HG conventional colposcopic impression, some were in a "conservative management" pathway, for whom a repeat colposcopy was advised. In contrast to previous studies, where biopsies had to be taken from positive areas of the DSI map $[4,5]$, in routine practice the use of the DSI map for biopsy will inevitably vary, as colposcopic practice varies. This may explain why some HG indications on the DSI map were not biopsied (Table 4). A review of the followup of the women who had no biopsies, or biopsies showing CIN1 or less at 3 years is planned and will be the subject of future studies.

Furthermore, the total number of biopsies taken was not recorded, and so we cannot verify whether an improved detection with use of the DSI map could be explained simply by a more extensive sampling. Also, the differing biopsy rates between clinics cannot be explained by the proportion of HG referrals. This was despite a threshold approach for the DSI map to indicate a need and the site of biopsy. The final histological outcome was correlated with the prediction of conventional or DSI colposcopy. This did not depend on whether biopsies were taken before or after the DSI mapping.

Eight of 10 CIN2+ cases thought to be normal at conventional colposcopy were identified by the DSI map from our service evaluation of 393 patients. Extrapolating this to all-Wales and English practice, 8,752 new patients (abnormal cytology and other) were seen in Wales in 2014 (CSW 1.4.13-31 [21] and 199,322 were referred in the same period in England (Cervical Screening Program England 2014-2015) [22]. So 178 women/year would have CIN2+ diagnosed in Wales as a consequence of the DSI map when conventional colposcopy identifies a normal cervix. This would be 4,057 women in England.

It is expected that, as with colposcopy in general, the DSI map would be less useful when the transformation zone is incompletely visualized (a type 3 transformation zone). Among the study population, 5\% had a type 3 transformation zone and were consistent with all-Wales data. The DSI map has not been evaluated previously for the identification of vaginal extension of CIN and was not used for vaginal examination in this service evaluation.

Both of the early clinical studies on DSI colposcopy found a statistically significant higher sensitivity for identifying CIN2+ women compared to conventional colposcopy $[4,5]$. It is worth noting that in both studies all women had biopsies to minimize the verification bias. A study of 239 women from Denmark reported that the DSI map failed to impact CIN2+ detection significantly. According to the authors, the assessment was done with the outcomes analyzed in the reverse order than here, previously and in clinical practice, raising concerns about the methodology, which the authors also acknowledge [23]. These findings have not been reproduced elsewhere.

The Welsh screening program did not use HPV triage of LG abnormalities at the time of service evaluation, and the HPV status of the participating patients is unknown. In the longer term, the consequences of HPV-based screening may be to reduce sensitivity for colposcopy as more women are expected to be referred without a significant lesion and the incidence of CIN3+ has been shown to be reduced with continued screening rounds [24-26]. Overall, alternative colposcopic technologies should be considered to improve the sensitivity of detecting CIN2+ in HPV-based screening programs.

The current service evaluation is a multi-center assessment of the performance of colposcopy with the DSI map in routine clinical practice, including patients referred with LG cytological results. Colposcopy with the DSI colposcope resulted in improved sensitivity to detect CIN2+ and maintained a high NPV for all referrals and especially for those with LG referral cytology.

\section{Acknowledgments}

M/s. S. Ashman, Nurse Specialist, University Hospital of Wales; M/s. J. Beattie, Nurse Specialist, Betsi Cadwaladr University Health Board; H. Clayton, Head of Information, CSW; EM Delicha, Biostatistics Consultant; Prof. A.N. Fiander, Consultant Gynecological Oncologist, University Hospital of Wales; R. Jones, Manager, CSW; Mr. K. Lim, Consultant Gynecological Oncologist, University Hospital of Wales; Dr. G.C. Rieck, Consultant Obstetrician and Gynecologist, Ysbyty Gwynedd; M/s. C. Robertson-Cowell, Llantrisant Hospital; M/s. J. Smith Nurse Specialist, Betsi Cadwaladr University Health Board; Mr. S. Watermeyer, Consultant Obstetrician and Gynecologist, Llantrisant Hospital. 
No funding was required to carry out this service evaluation. Loan of the DSI colposcopes was free of charge from DYSIS Medical.

\section{Ethical Approval}

Ethics approval was not required. All patients consented for colposcopy as per standard clinical practice in Wales.

\section{Disclosure Statement}

E.P. and A.D. are full-time employees of DYSIS Medical, the other authors declared no conflict of interest. DYSIS Medical paid the publisher fee.

\section{Funding Source}

No funding was provided for this paper.

\section{References}

1 Pretorius RG, Zhang WH, Belinson JL, Huang MN, Wu LY, Zhang X, Qiao YL: Colposcopically directed biopsy, random cervical biopsy, and endocervical curettage in the diagnosis of cervical intraepithelial neoplasia II or worse. Am J Obstet Gynecol 2004;191: 430-434.

2 Jeronimo J, Schiffman M: Colposcopy at a crossroads. Am J Obstet Gynecol 2006;195: 349-353.

3 Bekkers RL, van de Nieuwenhof HP, Neesham DE, Hendriks JH, Tan J, Quinn MA: Does experience in colposcopy improve identification of high grade abnormalities? Obstet Gynecol Survey 2009;64:97-98.

4 Soutter WP, Diakomanolis E, Lyons D, Ghaem-Maghami S, Ajala T, Haidopoulos D, Doumplis D, Kalpaktsoglou C, Sakellaropoulos G, Soliman S, Perryman K, Hird V, Buckley CH, Pavlakis K, Markaki S, Dina R, Healy V, Balas C: Dynamic spectral imaging: improving colposcopy. Clin Cancer Res 2009; 15: 1814-1820.

5 Louwers JA, Zaal A, Kocken M, Ter Harmsel W, Graziosi G, Spruijt J, Berkhof J, Balas C, Papagiannakis E, Snijders P, Meijer C, van Kemenade F, Verheijen R: Dynamic spectral imaging colposcopy: higher sensitivity for detection of premalignant cervical lesions. BJOG 2011;118:309-318.

6 Zaal A, Louwers J, Berkhof J, Kocken M, ter Harmsel WA, Graziosi GC, Spruijt JW, Balas C, Papagiannakis E, Snijders PJ, Meijer CJLM, van Kemenade FJ, Verheijen RH: Agreement between colposcopic impression and histological diagnosis among human papillomavirus type 16-positive women: a clinical trial using dynamic spectral imaging colposcopy. BJOG 2012;119:537-544.

7 Gage JC, Hanson VW, Abbey K, Dippery S, Gardner S, Kubota J, Schiffman M, Solomon D, Jeronimo J; ASCUS LSIL Triage Study (ALTS) Group: Number of cervical biopsies and sensitivity of colposcopy. Obstet Gynecol 2006;108:264-272.

8 Brotherton JM, Fridman M, May CL, Chappell G, Saville AM, Gertig DM: Early effect of the HPV vaccination programme on cervical abnormalities in Victoria, Australia: an ecological study. Lancet 2011;377:20852092.

9 Department of Health. Annual HPV Vaccine Coverage in England: 2012-2013. https:// www.gov.uk/government/uploads/system/ uploads/attachment_data/file/266207/HPV_ AnnualDataTable2012_13_AT_acc5.pdf (accessed February 15, 2014).

10 Public Health Wales. Latest Quarterly Report for the Uptake of Routine Childhood Immunisations in Wales. 2013. http://www.wales. nhs.uk/sitesplus/888/news/29039 (accessed February 15, 2014).

11 Van Effelterre TP, Hogea C, Taylor SM: Projected impact of Cervarix ${ }^{\mathrm{TM}}$ vaccination on oncogenic human papillomavirus infection and cervical cancer in the United Kingdom. Hum Vaccin Immunother 2016;12:8-19.

12 Zorzi M, Del Mistro A, Farruggio A, de'Bartolomeis L, Frayle-Salamanca H, Baboci L, Bertazzo A, Cocco P, Fedato C, Gennaro M, Marchi N, Penon MG, Cogo C, Ferro A: Use of a high-risk human papillomavirus DNA test as the primary test in a cervical cancer screening programme: a population-based cohort study. BJOG 2013;120:1260-1267.

13 Cervical Screening Wales. Annual Statistical Report 2014-2015. 2015. https://www.cervicalscreeningwales.wales.nhs.uk/statistical-reports (accessed June 22, 2017).

14 Schiffman M, Wentzensen N, Wacholder S, Kinney W, Gage JC, Castle PE: Human papillomavirus testing in the prevention of cervical cancer. J Natl Cancer Inst 2011;103:368-383.

15 Coronado PJ, Fasero M: Colposcopy combined with dynamic spectral imaging. A prospective clinical study. Eur J Obstet Gynecol Reprod Biol 2016;196:11-16.

16 NICE: Adjunctive Colposcopy Technologies for Examination of the Uterine Cervix - DySIS and the Niris Imaging system. National Institute for Health and Clinical Excellence. Diagnostic consultation document. 2012. http://publications.nice.org.uk/adjunctivecolposcopy-technologies-for-examinationof-the-uterine-cervix-dysis-and-the-nirisdg4 (accessed March 30, 203).

17 Louwers JA, Zaal A, Kocken M, Berkhof J, Papagiannakis E, Snijders PJ, Meijer CJ, Ver- heijen RH: The performance of dynamic spectral imaging colposcopy depends on indication for referrals. Gynecol Oncol 2015;139:452-457.

18 Fay MP: Two-sided exact tests and matching confidence intervals for discrete data. R Journal 2010;2:53-58.

19 Cervical Screening Wales. KC53/61/65 Statistical Report - Adroddiad Ystadegol 20122013. 2014. http://www.screeningservices. org.uk/csw/prof/reports/KC53-61-65_1213.pdf (accessed January 28, 2014).

20 Health and Social Care Information Centre. Cervical screening programme, England. Statistics for 2014-2015. 2015. http://content. digital.nhs.uk/catalogue/PUB18932/nhscervical-stat-eng-2014-15-rep.pdf (accessed June 20, 2017).

21 KC53/61/65 Statistical Report - Cervical Screening Wales 2013/14. www.cervical screeningwales.wales.nhs.uk/opendoc/ 248029 (accessed January , 2018).

22 Cervical Screening Programme, England 2014-2015. NHS Digital. https://digital.nhs. uk/catalogue/PUB18932. (accessed January 26,2018 )

23 Roensbo MT, Hammer A, Blaakær J: Can dynamic spectral imaging system colposcopy replace conventional colposcopyin the detection of high-grade cervical lesions? Acta Obstet Gynecol Scand 2015;94:781-785.

24 Arbyn M, Ronco G, Meijer CJ, Naucler P: Trials comparing cytology with human papillomavirus screening. Lancet Oncol 2009;10:935-936.

25 Anttila A, Kotaniemi-Talonen L, Leinonen M, Hakama M, Laurila P, Tarkkanen J, Malila N, Nieminen P: Rate of cervical cancer, severe intraepithelial neoplasia, and adenocarcinoma in situ in primary HPV DNA screening with cytology triage: randomised study within organised screening programme. BMJ 2010;340:c1804.

26 Katki HA, Kinney WK, Fetterman B, Lorey T, Poitras NE, Cheung L, Demuth F, Schiffman M, Wacholder S, Castle PE: Cervical cancer risk for women undergoing concurrent testing for human papillomavirus and cervical cytology: a population-based study in routine clinical practice. Lancet Oncol 2011;12:663672 